\title{
Belgeo
}

Revue belge de géographie

4 | 2018

Transformations of urban space after the fall of Socialism

\section{De la ville pionnière à la cité}

Les nouveaux paysages des villes pétrolières de la plaine de l'Ob

From frontier town to modern city: the new landscapes of oil and gas towns in the $\mathrm{Ob}$ river plain

\section{Yvette Vaguet}

\section{(2) OpenEdition}

1 Journals

\section{Édition électronique}

URL : http://journals.openedition.org/belgeo/24777

DOI : 10.4000/belgeo.24777

ISSN : 2294-9135

Éditeur :

National Committee of Geography of Belgium, Société Royale Belge de Géographie

Référence électronique

Yvette Vaguet, « De la ville pionnière à la cité », Belgeo [En ligne], 4 | 2018, mis en ligne le 18 juillet 2018, consulté le 09 octobre 2020. URL : http://journals.openedition.org/belgeo/24777 ; DOI : https:// doi.org/10.4000/belgeo.24777

Ce document a été généré automatiquement le 9 octobre 2020.

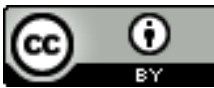

Belgeo est mis à disposition selon les termes de la licence Creative Commons Attribution 4.0 International. 


\title{
De la ville pionnière à la cité
}

\author{
Les nouveaux paysages des villes pétrolières de la plaine de l'Ob \\ From frontier town to modern city: the new landscapes of oil and gas towns in \\ the $\mathrm{Ob}$ river plain
}

Yvette Vaguet

1 En attendant l'économie décarbonée, les hydrocarbures continuent de structurer la géographie et la géopolitique du monde. Pour autant, parfois à la limite de l'œcoumène, les lieux d'extraction et les modes de vie des personnes s'activant à tirer ces précieux produits des sous-sols retiennent somme toute assez peu l'attention. Nous considérerons ici la vaste plaine marécageuse arctique et subarctique de Sibérie occidentale laquelle a été brutalement urbanisée durant les dernières décennies du régime communiste. Que deviennent ces lieux depuis l'effondrement du régime qui les avaient commanditées? Cette contribution, largement appuyée par l'image à différentes échelles, présente un éclairage sur les transformations des paysages urbains post-soviétiques, situés sur les marges glacées de l'humanité.

\section{Contexte}

2 La région d'intérêt, la plaine de l'ob, est ici réduite aux deux districts autonomes (okroug) producteurs d'hydrocarbures: celui de Khantys-Mansis sur l'ob moyen, produisant surtout du pétrole, et celui de Iamalo-Nenetsie sur l'ob inférieur, bordant l'océan Arctique et présentant une prédominance de la production gazière (figure 1). Ces deux okroug sont sous la juridiction d'un autre sujet de la Fédération de la Russie, l' oblast méridional de Tioumen, lequel borde le Kazakhstan et se trouve exclu de l'étude. $\mathrm{Au}$ total, la zone de recherche couvre une superficie supérieure à 1,3 millions de $\mathrm{km}^{2}$, soit près du tiers de l'Union Européenne. Or, à ces latitudes et en arrière des monts Oural qui bloquent les masses d'air océanique, la majorité du sol est gelé en permanence, largement de façon continue et sur une épaisseur plurimétrique.

Connue historiquement comme l'espace d'autochtones nomades, principalement cueilleurs-chasseurs-pêcheurs (comme les Khantys et les Mansis), ou éleveurs de 
rennes dans la partie plus septentrionale (comme les Nenets), la région expérimenta à partir des années 1960, une transition territoriale fondée sur le développement industriel et urbain. De fait, le régime soviétique a multiplié les villes qui furent un outil de la planification économique du pays. Actuellement, les $2 / 3$ des villes de la Russie ont été fondées après 1917 (Chatel, Moriconi-Ebrard, 2007 ; Motta, 2007). La transition régionale a été conduite rapidement sur les marges de l'œcoumène, faisant reculer l'érème, son complément sauvage (Berque, 2011). Il s'agit là bel et bien d'un front pionnier défini comme une « région de lisière » que « l'occupation sédentaire des hommes n'a pas encore conquise » (Demangeon, 1932). Toutefois, celui-ci est discontinu et industriel; les villes, une fois établies, devenaient bases-arrière de nouveaux établissements humains plus au nord, d'abord dans la taïga, puis dans la toundra, enfin au-delà du cercle arctique depuis les années 1980. Au total, 23 établissements humains sont urbains dont 21 ont obtenu le statut de ville (gorod) après 1960. Cette conquête spatiale a grandement été alimentée par des apports migratoires exogènes à la région ${ }^{1}$ si bien que les rapports démographiques et économiques ont été renversés entre l'oblast méridional (sur le transsibérien) et les districts septentrionaux. Ceux-ci furent rapidement intégrés au reste $\mathrm{du}$ bloc communiste et devinrent un des bassins d'hydrocarbures les plus vastes du monde, contribuant largement à placer de façon stable la Russie parmi les premiers pays producteurs (Marchand-Vaguet, 2005).

Figure 1. La Sibérie occidentale hier et aujourd'hui.

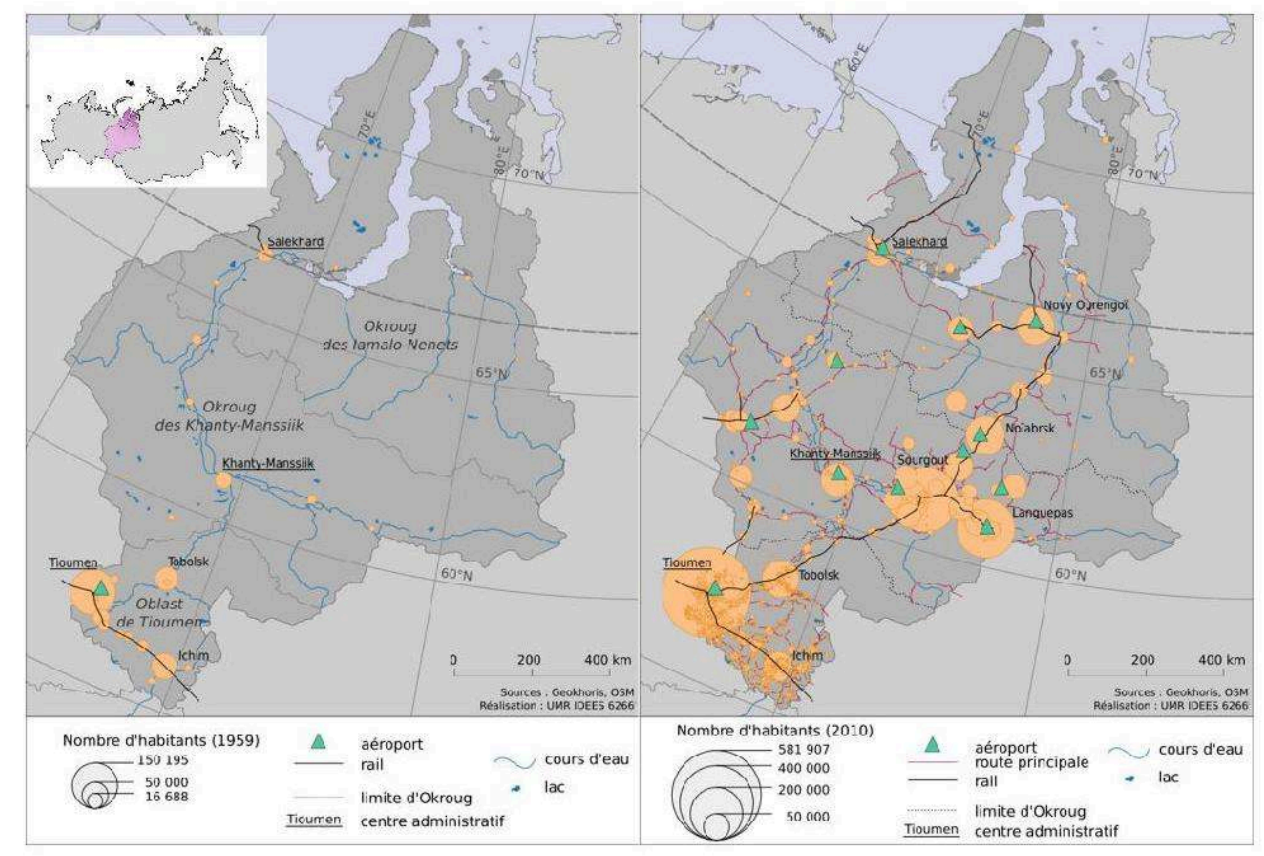

Comment les sciences géographiques ont-elles caractérisé ce type d'espace en général et en quoi les concepts classiques correspondent-ils à la plaine de l'ob en particulier ? F.J. Turner, historien et sociologue, pose le premier l'expression de frontier dans sa thèse intitulée The Frontier in American History en 1893 (Juricek, 1966). Traduit par front pionnier, le concept est d'emblée historique, associé à toute l'Histoire d'une nation et au-delà, selon son ampleur, à celle de l'humanité. Sa dimension spatiale vient après (Demangeon, 1932). P. Monbeig applique ce concept aux plateaux caféiers paulistes, au Brésil (Monbeig, 1952, 1954; Robequain, 1952). Il finit d'ancrer pour certains le front 
pionnier dans le monde agricole et étudie le rôle des acteurs. Mais surtout, le front pionnier comporte une finalité géopolitique. Ainsi, au Brésil, agricole, il vise autant à abaisser les tensions agraires qu'à faire l'économie d'une réforme agraire, tout en intégrant des pans entiers du territoire. En Sibérie occidentale, industriel, il vise autant à faire entrer des pétrodollars pour le développement de l'appareil industriel soviétique encore en développement, qu'à fournir l'énergie nécessaire à celui-ci, et poursuivre la conquête territoriale encore inachevée (De Koninck, 2000 ; MarchandVaguet, 2005 ; Radvanyi, 2010). De plus, les dures épreuves qu'endurent les pionniers constituent une caractéristique commune à tous les fronts pionniers. A. Demangeon (1932) évoque "un combat opiniâtre " où " progressent lentement les pionniers de l'humanité ", à un rythme irrégulier. Cette lutte est celle de la civilisation contre la nature-sauvage de l'érème et elle est exacerbée en Sibérie occidentale par un environnement naturel extrême. Pour autant, le fait que la conquête s'y soit inscrite dans la seconde moitié du XXe siècle ajoute la variable «vitesse », notamment avec le recours à l'avion, qui explique le rapide processus d'urbanisation en comparaison avec des temps plus anciens.

5 Les villes de la plaine de l'ob ont vu le jour par la volonté du pouvoir central alors soviétique. Il n'est pas du tout certain qu'elles auraient vu le jour dans un système capitaliste considérant les coûts considérables (acheminement des denrées, coûts sanitaires, de construction...) liés à l'implantation permanente d'un peuplement humain dans les zones inhospitalières de l'Arctique. D'ailleurs, au Canada et aux EtatsUnis, depuis la seconde moitié du XXe siècle, avec la révolution des transports aériens, le choix est celui de favoriser les équipes de travail par rotation plutôt que l'habitat permanent. Cette posture vient aussi de l'expérience de la ruée vers l'or de la Klondike qui ouvrit un front pionnier en 1896. L'histoire a montré le coût élevé de gestion des villes fantômes, abandonnées lorsque la ressource est épuisée ou concurrencées par de nouveaux espaces de production.

6 Les jeunes villes pionnières ouest-sibériennes ont été confrontées à une rude transition économique et politique comme toutes leurs homologues soviétiques, mais sans doute pour elles les enjeux touchaient leur durabilité même. Comment ces villes allaient-elles survivre alors qu'elles étaient désormais soumises aux aléas macro-économiques (notamment le prix des hydrocarbures), au risque d'épuisement des réserves, à l'effacement d'une volonté politique de les soutenir, sans parler de leur vulnérabilité face aux risques naturels dans un environnement hostile et changeant (menaces d'inondation ou/et d'effondrement du pergélisol liées au changement climatique) ? A la question de la pertinence de maintenir ces villes à la limite de l'extrême dans une économie de marché, s'ajoute l'ouverture à la concurrence entre elles pour maintenir leur attractivité et diversifier leur profil mono-économique. Les programmes de développement socio-économique des villes de la plaine de l'Ob rendent compte de ces nouvelles préoccupations dont les réalisations se lisent dans le paysage urbain. Que sont devenues ces villes, parfois à la limite de la viabilité dans une économie de marché ? Il s'agit de discuter ici des transformations internes de ces villes soviétiques depuis les années 1990 et de mettre en évidence une certaine forme de normalisation définie comme processus de rapprochement avec la ville des économies de marché engagée dans la mondialisation telle que définie par G. Valentine (2001), pour qui chaque établissement urbain est engagé dans une féroce concurrence mondiale. 


\section{L'image comme outil}

7 Toutes les villes pionnières évoquent, dans leurs architectures et leurs modes d'habiter, la rudesse de la lutte pour "civiliser" l'espace sauvage comme le rapportent les écrivains (Jack London, L'appel de la forêt,...) et les cinéastes (Charlie Chaplin, La ruée vers l'or). L'image, qu'elle soit une vue de l'espace ou une vue du sol, constitue un outil efficace de relevé de l'inscription des hommes sur la terre. Elle est un moyen de classer et comparer dans l'espace et dans le temps. Les deux vues offrent de plus des focales très différentes qui répondent au besoin de changement d'échelle du géographe, lequel conduit d'incessants allers-retours entre les processus à des échelles fines et à des échelles plus englobantes. Ensuite, elle est utilisée comme médium pour rendre compte et soutenir l'argumentation du chercheur, sensible à ses usages, qu'elle soit spatiale ou prise à hauteur du promeneur sur le terrain.

8 L'image en tant qu'impression de la lumière « atteste de l'existence (mais non du sens) d'une réalité » (Berthomiere, 2012). L'image constitue un bon support d'une approche par le paysage rendant compte des mutations de l'habiter de ces villes qui furent un temps à la frange septentrionale de l'œcoumène du plus vaste pays communiste. Cette démarche participe de la "nouvelle " Géographie Urbaine qui se caractérise par le souci de chercher à lier le matériel et l'immatériel (Lees, 2002). Deux approches qui ne sont plus (enfin) nécessairement mutuellement exclusives et le courant actuel de "rematérialisation" apparaît comme un moyen de cibler un équilibre entre ces deux pensées (Latham \& McCormack, 2004). La contribution de l'image y a toute sa place parce qu'elle rapporte l'expression matérielle des phénomènes et processus qui s'exercent au sein des territoires.

9 Cette approche phénoménologique appuyée par la combinaison de l'image et du texte forme un message bi-media (Moles, 1978). L'analyse appelle donc une intimité avec le terrain, ici développée au fil de nombreuses missions, quasi-annuelles, depuis 1997. Le dialogue entre les vues spatiales et les vues au sol assure les changements de focales, et favorise toute "la démarche géographique [qui] vise la compréhension de ce qui se passe dans le lieu, à travers les modes d'habiter des habitants qui le pratiquent au quotidien, l'aménagent» (Biaggi, 2015). L'habiter se concentre sur les usagers de l'espace; les pratiques individuelles et collectives sont privilégiées car elles donnent accès aux valeurs, aux aspirations qui font des habitants des " actants ", c'est-à-dire des résidents dotés d'une capacité d'action, de prise en main de leur territoire. C'est ainsi que le front pionnier agricole brésilien, avec ses acteurs, a été abordé par l'imagerie spatiale (Selleron, 2004). L'image donne accès à la trace objective que les usagers laissent dans l'espace - géo-graphier.

Les paysages urbains en plaine de l'ob portent des marqueurs du processus d'urbanisation communiste à la frontier et des transformations post-soviétiques. Il s'agit d'analyser par l'image, leurs mutations révélant celles de leurs modes d'habiter. Pour ce faire, nous retenons deux facettes complémentaires de la vie du résident : l'espace public et l'espace privé. A celles-ci nous ajoutons les constructions à vocation explicite de landmark et les espaces de consommation et/ou de récréation, qui découlent de l'injonction de rendre la ville attractive. 


\section{L'espace public}

11 Plusieurs points sont à souligner à l'échelle de la ville pour ce qui relève de l'espace public. Ce que le promeneur expérimente au premier chef est le niveau de verdure de la ville. Ces villes pionnières établies rapidement pour servir l'appareil productif, l'ont été au milieu de la taïga, plus rarement de la toundra. Elles semblent avoir été le fait de constructions édifiées au cœur de cette vaste forêt sans trop que celle-ci ait été essartée au préalable. En témoignent, l'omniprésence des arbres, largement des bouleaux, qui prospèrent sur le sol sablonneux de la plaine de l'ob (figure 2). Aujourd'hui, sans disparaître, ces éléments du paysage urbains deviennent toutefois de plus en plus morcelés, réduits à des paillettes laissées entre le bitume qui vient maintenant jusqu'aux entrées des immeubles.

Figure 2. Des villes pionnières au cœur de la taïga.
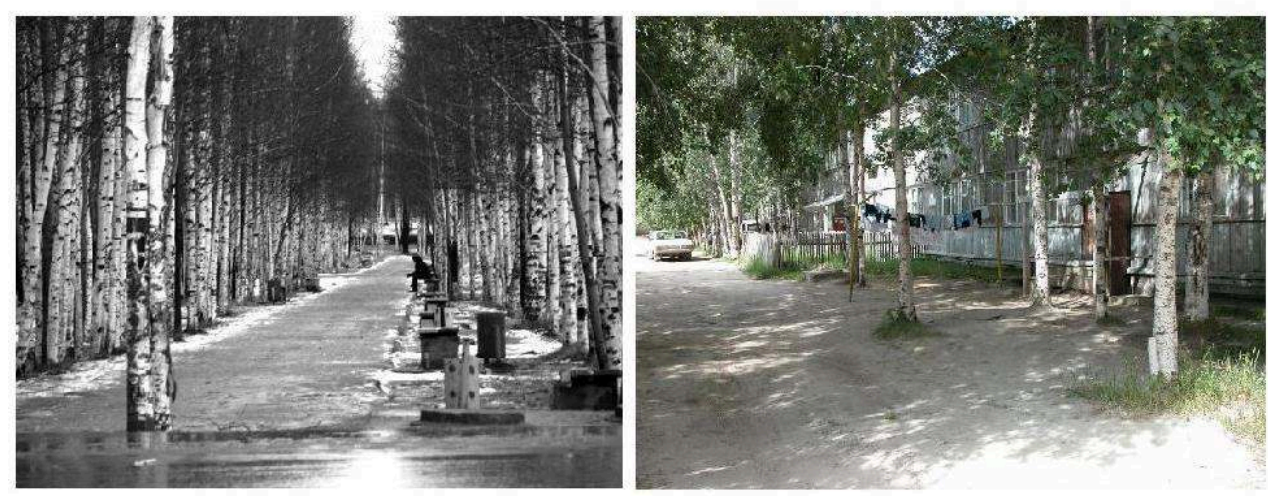

A gauche : Nefteïougansk, $61^{\circ} 06^{\prime} \mathrm{N} 72^{\circ} 36^{\prime} \mathrm{E}$, pétrole, ville depuis 1967, 102000 hab. (hiver 2000) - A droite : Noïabrsk, $63^{\circ} 11^{\prime} \mathrm{N} 75^{\circ} 27^{\prime} \mathrm{E}$, pétrole surtout et gaz aussi, ville depuis 1982, 109000 hab. (été 1999).

(C) photos: Y. Vaguet

12 Le second élément proéminent que ne peut manquer de noter l'observateur relève des caractéristiques du plan de ville soviétique. En premier lieu, le plan en damier et le zoning avec une zone industrielle séparée de la zone résidentielle et l'organisation en grands ilots bordés par de grandes artères de circulation. L'ilot, le micro-raïon, constitue l'unité fonctionnelle élémentaire de la ville soviétique. Il se compose d'immeubles de logements sur son pourtour et de bâtiments en son cœur dédiés aux services de la vie quotidienne de sa population (jardins d'enfants, crèches, écoles, cantines, équipement commercial de proximité, aires de sport et jardins). Or, ceux-ci ont toujours grandement manqué dans ces villes pionnières et si des efforts sont effectués avec la construction de complexes sportifs par exemple, le compte n'y est pas (figure 3). Ces grands traits de la structuration de l'espace urbain perdurent; néanmoins des changements importants se sont opérés. 
Figure 3. Urbanisme soviétique et transformations, vues du ciel.
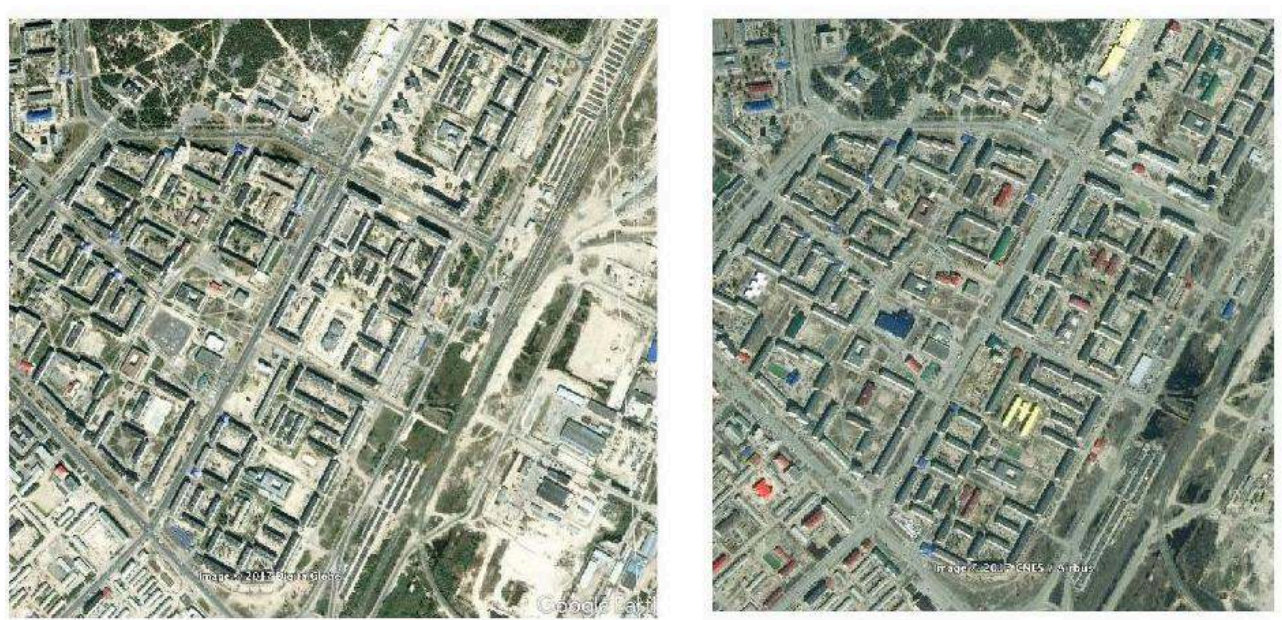

Noïabrsk, $63^{\circ} 11^{\prime} \mathrm{N} 75^{\circ} 27^{\prime} E$, pétrole surtout et gaz aussi, ville depuis 1982, 109000 hab. Zone résidentielle à l'ouest de la grande artère ; zone industrielle à l'est. On notera la présence du sol sableux partout, et des immeubles récemment coiffés de toits colorés.

(c) Google Earth (à gauche : août 2004 fin de l'été ; à droite : avril 2014 / fin de l'hiver)

Figure 4. Arrivée de la couleur, disparition des fils électriques - vues au sol.
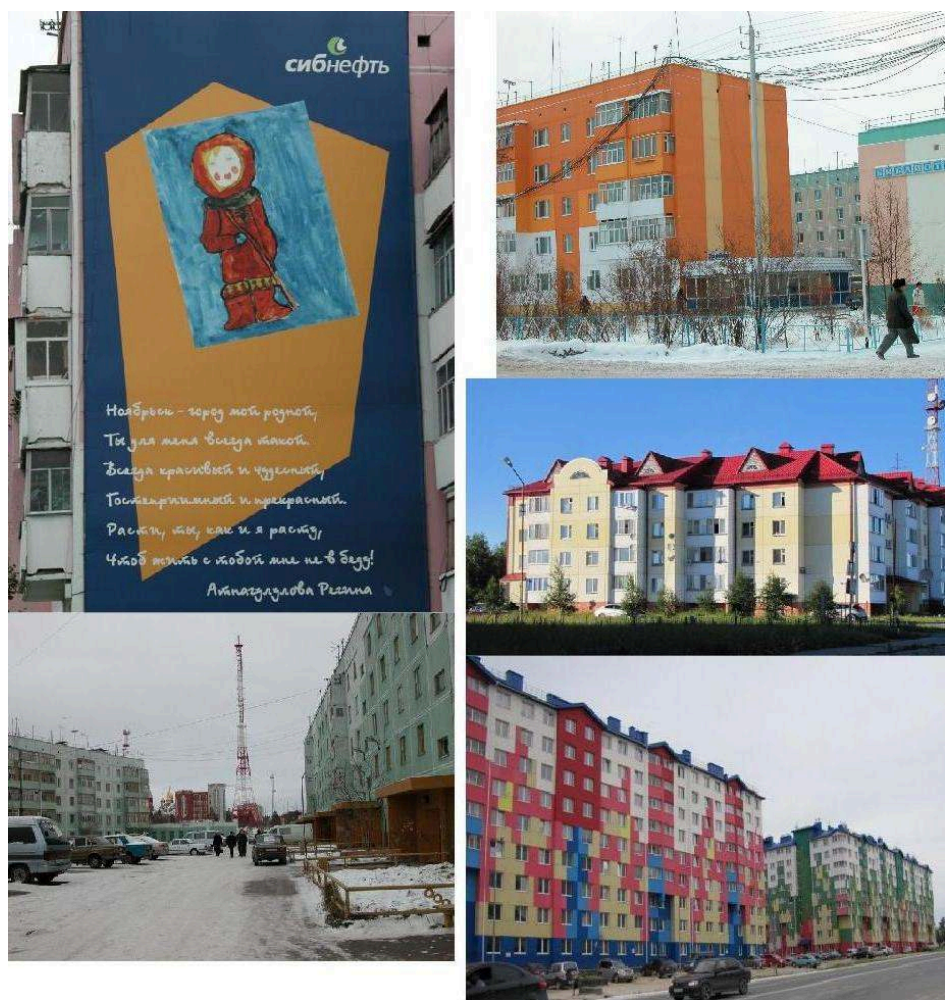

Noïabrsk, $63^{\circ} 11^{\prime} \mathrm{N} 75^{\circ} 27^{\prime} \mathrm{E}$, pétrole et gaz, ville depuis 1982, 107000 hab. Mouravlenko, $63^{\circ} 46^{\prime} \mathrm{N}$ $74^{\circ} 31^{\prime} E$, pétrole, ville depuis 1990, 37000 hab. A gauche : les rares immeubles peints l'étaient par la compagnie pétrolière Sibneft, ils étaient encore visibles au début des années 2000, tout comme les innombrables fils électriques dans la ville. (2002 \& 2003). A droite : la couleur est apparue à la faveur d'opérations de rénovation : en haut, à Mouravlenko en 2008, les fils électriques sont encore très présents ; au milieu : les immeubles ont été ensuite coiffés de toits colorés, et de nouvelles constructions ; en bas, à Noïabrsk en 2015 les fils électriques disparaissent.

(C) photos : Y. Vaguet 
La nature des constructions a évolué. Dans un premier temps, la construction des logements n'était pas prioritaire par rapport à l'industrie et beaucoup de résidents se trouvaient logés dans des dortoirs en bois. Ceux-ci demeurent fonctionnels et sont le fait de quartiers réguliers composés d'immeubles longs de 35 à 55 mètres. Leurs habitants sont des néo-résidents dans la ville ou des travailleurs de quart; ils constituent la classe sociale la plus défavorisée (figure 5). D'une façon générale, les constructions en bois sont encore largement répandues ; néanmoins elles disparaissent, moins par la volonté de l'aménageur que par des incendies et les habitants les évitent autant que possible. A Noïabrsk, la ville fût implantée d'abord sur une zone qui se révéla marécageuse (Noïabrsk-1) ; elle fût alors transférée quelque $12 \mathrm{~km}$ plus au nord (Noïabrsk-2). Aujourd'hui, le train s'arrête à deux gares mais le quartier de Noïabrsk-1 se compose toujours essentiellement de maisons de bois dans la forêt et le relogement des habitants s'éternise. Dans un second temps de la période soviétique, avec la maturation de la ville, les baraques de bois ou les tronçons de pipes aménagés des primo-arrivants ont côtoyé les immeubles typiquement soviétiques, surtout des khrouchtchevki, immeubles préfabriqués comptant 5 étages. Du reste, ces constructions, pensées à Moscou et multipliées sur tout le territoire national sans adaptation locale, souffraient ici de la rudesse du climat (figure 4). A partir de la fin de la décennie 1990, la transition économique s'est illustrée par la diversification des constructions, donnant à lire dans le paysage urbain des disparités sociales peu visibles jusqu'alors. Une diversité également renforcée par l'irruption de la couleur. Lors de mes premières missions, l'espace public était encore très peu coloré. Je me souviens d'un long terrain à l'hiver 2000 ; je fus frappée (au retour) par le sentiment d'avoir vécu en noir et blanc durant plusieurs mois - certes une impression renforcée par la nuit polaire, la neige et l'écorce des bouleaux effeuillés, néanmoins, les seules couleurs arborées par les immeubles étaient les quelques peintures à l'effigie des industries extractives vantant la ville. Depuis, les restaurations se sont multipliées et de nouveaux immeubles et les cottages, portent des couleurs nombreuses (trop ?) et des formes originales (figures 3 et 4). 

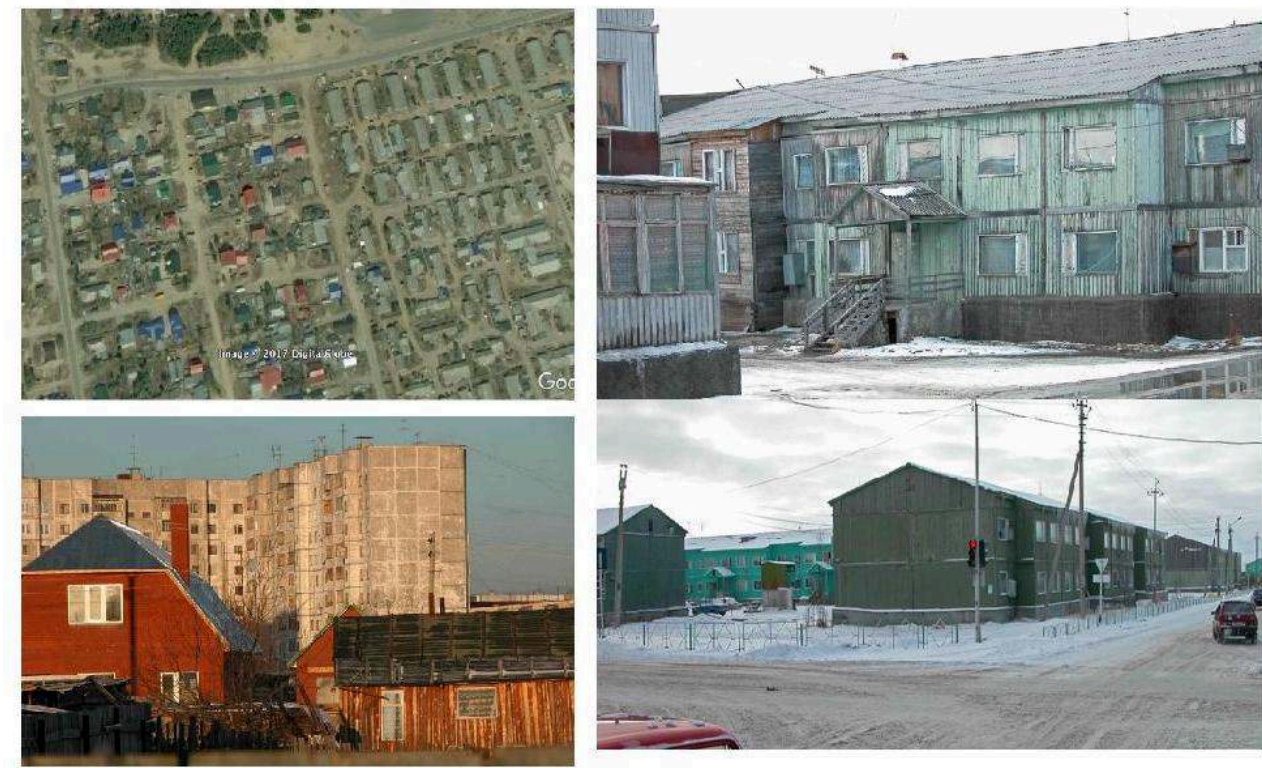

En haut à gauche : à l'est, un quartier de dortoirs jouxte à l'ouest, un quartier de résidences individuelles (avril 2005, Noïabrsk @ C Google Earth) ; en haut à droite : dortoirs à Noïabrsk ; en bas à droite : quartiers de dortoirs à Mouravlenko ; en bas à gauche : Sourgout, $61^{\circ} 15^{\prime} \mathrm{N} 73^{\circ} 24^{\prime} \mathrm{E}$, pétrole, ville depuis 1965, 282000 hab. La densification de la ville témoigne de la sédimentation des périodes de construction et des régimes politiques successifs (de la baraque de bois, puis l'immeuble soviétique et enfin au cottage individuel de brique post-soviétique).

(c) photos: Y. Vaguet

\section{Travailler son image dans un marché dorénavant concurrentiel}

14 La culture est le médium par lequel les villes expriment leur identité, leur caractère, leur singularité et cherchent à donner une image positive d'elles-mêmes. Par-là, elles affirment ce qu'elles souhaitent être, ce dont elles dépendent et où elles vont. Entrant dans une économie de marché, les villes soviétiques se trouvent dorénavant confrontées à une concurrence que l'on connait bien en Occident (Valentine, 2001). Dans la plaine de l'ob, les villes pionnières doivent désormais plus que jamais concourir pour attirer et maintenir les capitaux, les entreprises, et les habitants, surtout les plus qualifiés. Il en résulte qu'elles apprennent aussi à "se vendre", comme leurs homologues occidentales, à "glamouriser la ville». La conséquence en est la multiplication de landmarks, soit par l'architecture des sièges, au moins régionaux, des grandes compagnies qui affirment par là-même leur pouvoir, soit par des monuments suggestifs. Jadis, l'hélicoptère qui avait déposé les premiers pionniers et le premier train constituaient les monuments caractéristiques de la mémoire collective locale. Autrement dit, ils illustraient, voire stimulaient, la conquête, la situant implicitement toujours face à une nature sauvage à maîtriser, et surtout à maîtriser ensemble car il s'agissait bien de mettre en relief l'aventure collective. Aujourd'hui, ils font plus facilement référence aux deux côtés de la frontier, c'est-à-dire à l'idée d'une identité complexe et originale composée de l'autochtonie d'un côté, et de l'industrie de l'autre côté, donnant ainsi une épaisseur au front pionnier, au moins dans la représentation 
(figure 6). Dans tous les cas, il s'agit de construire une identité locale et stimuler l'attachement des habitants.

Figure 6. Emergence de nouveaux landmarks.
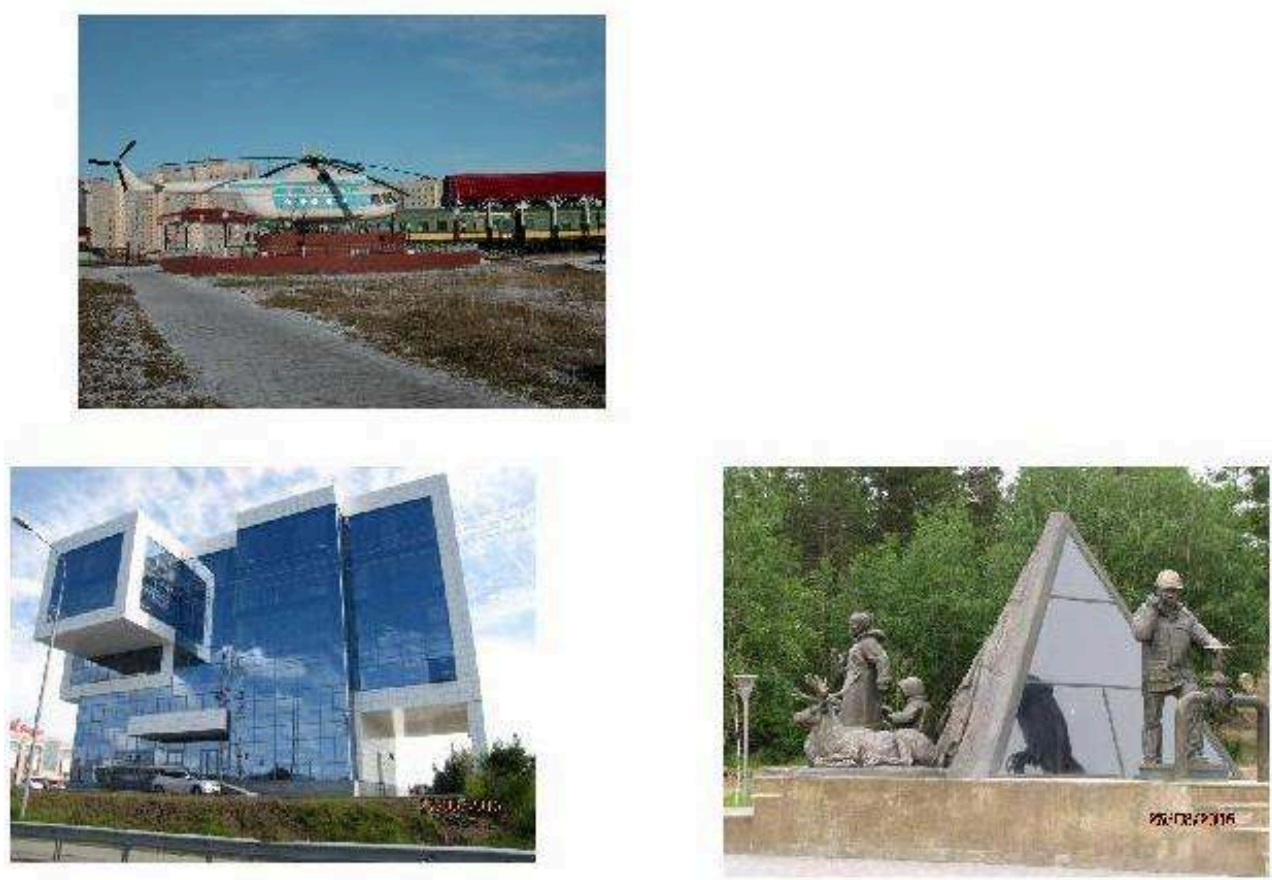

En haut : Les premiers monuments de la ville ont souvent été l'hélicoptère qui déposa les premiers colons et/ou le premier train (2002, Languepass) ; en bas à droite : les monuments plus récents témoignent moins de la conquête spatiale que de la quête d'une identité locale originale (2015, Noïabrsk) ; en bas à gauche : les compagnies privées utilisent aujourd'hui l'architecture comme marqueur de puissance (2015, Sourgout).

(c) photos: Y. Vaguet

\section{L'émergence de paysages de consommation et de récréation}

Les municipalités, lors de leur analyse SWOT $^{2}$ incluse dans leurs programmes de développement, n'hésitent pas à faire référence à la concurrence des autres villes proches, riches aussi de pétrole et/ou de gaz. C'est le cas de Noïabrsk qui cherche à affermir sa position parmi les villes du système régional. Cet établissement s'appuya sur Sourgout au sud, d'où partit le front pionnier. Ensuite, la cité pionnière devint à son tour la base-arrière de Mouravlenko et Goubkinski qui se développèrent jusqu'à devenir elles-mêmes des villes à part entière. Aujourd'hui, le schéma de développement de Noïabrsk jusqu'en 2020 (Noyabrsk, 2017) mentionne explicitement la compétition avec sa grande sœur, Sourgout, et le risque de compétition avec ses petites sœurs, Mouravlenko et Goubkinski où il y a déjà davantage d'espaces culturels et récréatifs. La concurrence inter-ville concerne au premier chef la population; comment la retenir, l'attirer et notamment les jeunes diplômés. De fait, les villes ont longtemps présenté un fort renouvellement de la population. Les apports migratoires associés à la transition spatiale ont apporté des résidents, souvent des néo-polaires, non adaptés à l'environnement arctique et sans lien affectif avec celui-ci. Ainsi, ils étaient toujours 
prêts à repartir, en l'occurrence plus au Nord, à la recherche d'un meilleur salaire et de meilleures conditions de vie (Vaguet, 2013). Une enquête conduite en 1987-1988 auprès de 4200 travailleurs de l'industrie extractrice dans quatre villes de la région montrait que les résidents restaient rarement plus de cinq ans dans le district le plus septentrional (Logunov, 1999). Mes quelque 26 interviews approfondies, conduites lors de terrains après la chute de l'URSS, montraient toujours une forte mobilité - fluidité pour reprendre le terme de A.N. Zaitseva (2002), bien que la fluidité désirée soit désormais entravée par les prix de transport revus à la hausse, autre conséquence de la libéralisation. Ainsi, en 2012, le site officiel de la mairie de Noïabrsk constatait amèrement la difficulté de retenir la population hautement qualifiée. Depuis le site n'en fait plus mention, non pas que la question ne soit plus d'actualité mais l'usage du site est devenu une vitrine, un outil de communication de la ville (Noyabrsk, 2017). De fait, toutes les villes russes, y compris en Arctique, développent, avec un succès variable, la pratique, généralisée à l'ouest depuis longtemps, du "branding ». C'est-àdire travailler l'image de la ville, à l'instar d'une marque commerciale, la "vendre », la présenter de façon positive afin de maintenir les résidents et d'attirer les investisseurs, voire comme des métropoles, d'attirer les grands évènements qui ont des retombées économiques non négligeables. Par exemple, Mourmansk met en avant son 100e anniversaire, Arkhangelsk se pose comme capitale du Nord et accueillera en 2020 le prochain International Congress on Arctic Social Sciences (ICASS X).

Ainsi, les municipalités, à l'instar de Noïabrsk, insistent dans leur programme de développement socio-économique sur la qualité de vie des résidents et les jeunes constituent la tranche d'âge tout particulièrement ciblée, et par voie indirecte leurs parents à risque de souhaiter partir, en quête d'une meilleure vie pour leurs enfants. Des améliorations de la qualité de vie sont donc opérées : on peut citer la construction de lieux d'éducation, car à la fin des années 1990 les enfants étaient encore scolarisés en deux groupes, ceux du matin et ceux de l'après-midi, une équation qui permettait de pallier le manque d'écoles, au moment où la population jeune présentait un fort taux de natalité. Des nouveaux complexes sportifs sont aussi prévus, y compris un aquaparc qui devra rayonner jusqu'à Goubkinski et Mouravlenko respectivement à 120 et $250 \mathrm{~km}$. De même, les transports et la santé forment des secteurs clefs ciblés ; une nouvelle clinique est prévue. Le retard était tel qu'il peut sembler que l'on soit plutôt dans le simple rattrapage pour, enfin, répondre aux attentes des habitants. Toutefois, pour réaliser de telles constructions modernes, les villes gazières et pétrolières de la plaine de l'ob jouissent sans doute de plus de moyens que leurs consœurs arctiques grâce aux richesses du sous-sol et ce en dépit du retrait graduel de la participation des compagnies extractives à leur budget. En effet, en conséquence de la transition économique, celles-ci tendent à resserrer leurs activités sur leur fonction première.

L'économie de marché stimule le développement de l'économie des services et notamment le commerce très déficient jadis. Dans des villes qui, plus que monofonctionnelles, étaient mono-entreprises et le demeurent encore largement, le petit entreprenariat devient florissant d'autant que l'Etat mène une politique favorable dans les régions $d u$ nord. Les petites boutiques se sont multipliées et, surtout, elles ont maintenant pignon sur rue tandis que les commerces étaient autrefois dans les immeubles, ce qui exigeait une grande connaissance de la ville pour la pratiquer. Les murs de la ville ont ainsi pris les couleurs de la publicité, laquelle était tout à fait absente encore au début des années 2000. De plus, l'ouverture du pays a conduit à 
l'introduction de grandes enseignes emblèmes de la mondialisation (Mac Donald's), parfois encore sous forme de simple point de conseil (IKEA) (figure 7).

Figure 7. Emergence des paysages de consommation.
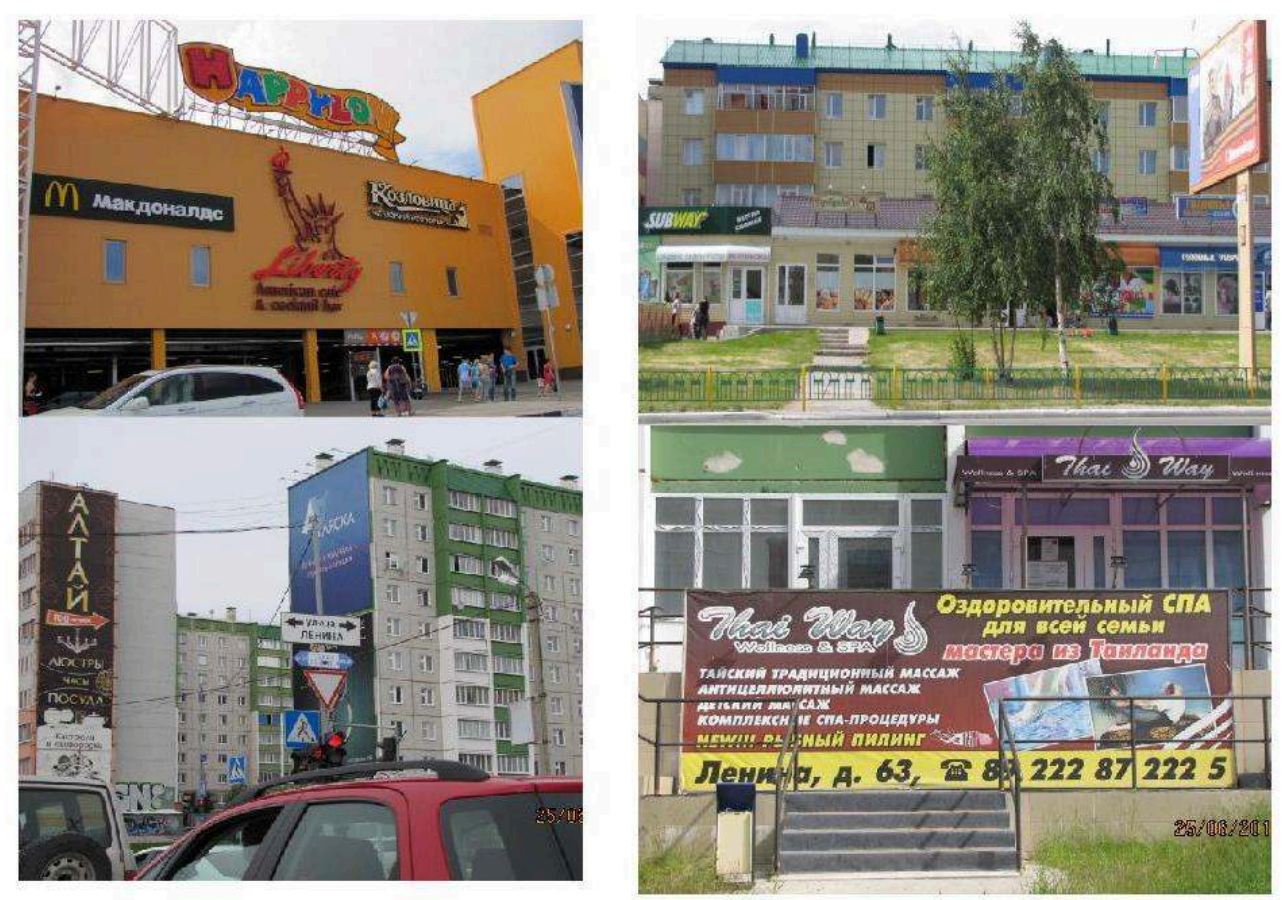

Depuis le coin haut gauche vers le coin bas droite : un centre commercial à Sourgout avec Mac Donald's; les commerces le long de la voie piétonne, au pied des immeubles à Noïabrsk avec Subway; un centre de massage thaï ouvert depuis peu; des grandes affiches publicitaires à la place des peintures de Sibneft.

(c) photos : Y. Vaguet, $2013 \& 2015$

Au total, émerge parmi les pionniers le flâneur, dépeint par Charles Baudelaire (1863), soulignant l'expérience sensible de la socialisation dans la foule et l'anonymat : « Pour le parfait flâneur, pour l'observateur passionné, c'est une immense jouissance que d'élire domicile dans le nombre, dans l'ondoyant, dans le mouvement, dans le fugitif et l'infini ». Pour autant, il ne s'agit pas d'un simple chaland: «le flâneur a un but plus élevé que celui d'un pur flâneur, un but plus général, autre que le plaisir fugitif de la circonstance. Il cherche ce quelque chose qu'on nous permettra d'appeler la modernité" (Baudelaire, 1863). Le flâneur, décrit comme piéton engagé dans une attitude moderne, est repris plus récemment en tant qu'actant dans la géographie de l'habiter (Valentine, 2001). Les centres commerciaux, les clubs et les restaurants constituent les trois types d'espace qui jouent un rôle clef dans ce processus de transformation. Ils participent à la mutation générale de la ville pionnière qui commence à ressembler aux autres cités modernes du monde. Finalement, ces villes post-socialistes voient un mode de vie urbain émerger en tant que façon de vivre dans un monde global, déjà loin des villes de pionniers à la frontière de l'œcoumène (Dybbroe, 2008 ; Dybbroe et al., 2010). 


\section{L'espace privé}

19 Dans l'économie collectivisée, la datcha constituait le seul îlot de propriété privée, certes le plus souvent modeste, sans chauffage ni eau courante. A la belle saison, les urbains qui en possédaient pouvaient quitter leur appartement pour le grand air et cultiver le lopin de terre associé afin de compléter notablement leur propre alimentation. Les logements étaient souvent communautaires, en Arctique la plupart du temps sous forme de dortoirs.

20 A l'inverse, la maison individuelle symbolise le modèle de développement occidental, l'économie de marché individualiste. Depuis la chute de l'URSS, la propagation de ce modèle dans l'espace post-soviétique a déjà été rapportée: «les nouveaux Russes continuent à se faire construire des luxueux cottages dans les terrains libres» (Le Huerou, 1998). D. Eckert (2001) notait de même la multiplication des résidences individuelles luxueuses dans Moscou en mutation dès la fin des années 1990. Toutes les grandes villes du pays ont été touchées par ce phénomène. Il relève d'une fraction riche de la population, ne lésinant pas sur les signes ostentatoires de richesse. Plus grandchose à voir donc avec la modeste datcha des parents et grands-parents de la période soviétique, si ce n'est que le « cottage» résulte parfois d'une profonde modernisation de la datcha.

21 Dans les villes arctiques et subarctiques, on aurait pu imaginer que ce modèle serait bloqué par les contraintes du milieu naturel et les longues distances rendant le coût d'acheminement des matériaux exorbitant. Pourtant, entre 1990 et 2000, les images satellites SPOT montrent clairement la multiplication des résidences particulières. Elles occupent, ici ou là, des interstices vacants, mais forment plus souvent de nouvelles extensions de la ville dans sa continuité (figure 5). Lors de mes derniers terrains, à Noïabrsk, j'ai pu entrer et observer les constructions d'un quartier résidentiel fermé $M e c h t a^{3}$, réservé aux classes sociales locales (très) privilégiées, à commencer par le maire (figure 8). Ce quartier dont l'entrée est contrôlée par un gardien rémunéré par les résidents, se situe à une vingtaine de kilomètres du cœur de la ville et s'étend sur 115 hectares.

Ce nouveau visage de la ville soviétique témoigne d'une volonté des résidents de quitter leur immeuble collectif pour une maison individuelle, souvent auto-construite, prenant la forme d'un cottage sophistiqué. Loin de la datcha, la richesse du propriétaire est exhibée, les deux types d'habitats n'ont en commun qu'une volonté de renouer avec la nature et d'assumer un certain art de vivre, avec "bagna", variante russe du sauna. Elle se localise en périphérie de la ville, de préférence autour des lacs, pour profiter de la wilderness (naturalité) sans crainte de la subir, comme c'était le cas des pionniers. 

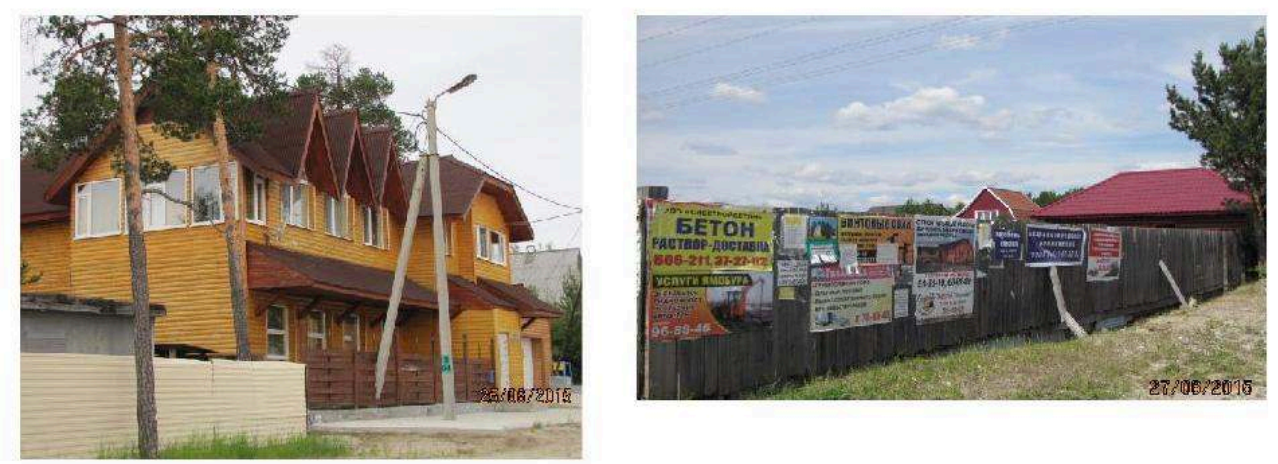

A gauche : Noïabrsk, la communauté fermée de Mechta à une vingtaine de kilomètre de la ville - à droite : Sourgout, quartier résidentiel fermé contigu à la ville et publicité de petites entreprises.

(c) photos: Y. Vaguet

\section{Conclusion - Discussion}

Tandis que les hydrocarbures structurent encore complétement nos modes de vie et nos mouvements sur la planète, nous ignorons le plus souvent comment les populations des industries extractives vivent et travaillent, parfois à la limite de l'œcoumène. A. Demangeon (1932) arguait que «l'occupation ne peut devenir permanente que si [les régions de front] se spécialisent dans une production de grande valeur ». Il est vrai que les hydrocarbures du sous-sol offraient à la région subarctique et arctique un atout en la matière. Toutefois, auraient-elles vu le jour dans une économie de marché à une période de l'Histoire où les transports aériens étaient déjà bien développés ? Rien n'est moins certain. L'environnement extrême et la productivité des puits baissant au moment de la chute de l'URSS auraient pu sonner leur fermeture d'autant que la transition allait de pair avec le désengagement de l'État sur le jeune front pionnier où la volonté politique et les moyens de la nation demeuraient encore cruciaux.

Pourtant, loin de leur démantèlement, les villes nouvelles de la plaine de l'ob se sont « urbanisées » si on osait, dans le sens où l'urbain n'est pas qu'une affaire de nombre d'habitants mais aussi une affaire de mode de vie (Collignon, 2008; Dybbroe, 2008 ; Dybbroe et al., 2010). De fait, il était autrefois possible d'évoquer "un peuplement urbain sans ville » (Vaguet, 2007a) dans ces lieux ayant obtenu le statut de ville en moyenne en 1983. De nouvelles strates, post-soviétiques, se sont depuis ajoutées dans l'urbanisme de ces villes qui sont devenues tout à fait modernes et contrastent étrangement au sein du Grand Nord russe. De fait, la plaine de l'ob, surnommée les émirats sibériens, a toujours présenté des salaires supérieurs à la moyenne nationale, permettant de maintenir son attractivité. Elle contraste encore avec la bordure septentrionale du pays qui a connu un exode dramatique dans sa globalité et plus particulièrement dans les régions les plus éloignées du quart nord-est: Magadan et Tchoukotka avaient perdu respectivement $53 \%$ et $67 \%$ de leur population entre 1989 et 2002 (Heleniak, 2003; Eckert, Jégou, 2004). L'argent des hydrocarbures circulant, combiné à un esprit d'entreprise, a permis à cette périphérie déjà intégrée mais toujours dépendante du centre, de valoriser elle-même une partie des capitaux locaux (Reynaud, 1981). C'est de cette vitalité dont témoignait un jeune diplômé de Moscou 
attiré par les opportunités de carrière dans une ville qui avait son âge, notait-il enthousiaste (Vaguet, 2007a).

Parler de «ville postsocialiste " revient à accréditer que toutes les villes de cette catégorie relèvent d'une identité commune, celle de ville "socialiste », et, que la chute de l'URSS les a fait entrer dans une phase de développement radicalement nouvelle (post) dont l'aboutissement implicite ou explicite est la ville globale, capitaliste (Coudroy de Lille, 2016). Les villes de la plaine arctique et subarctique de l'ob ont certes une identité "socialiste» mais celle-ci se mêle à une identité "pionnière " que partagent toutes les localités aux marges de l'œcoumène. Ainsi, leur trajectoire durant la période post-soviétique relève pour partie de la maturation « classique " d'un front pionnier qui se pérennise. Elles se métamorphosent peu à peu en villes comme lieu de vie avec un mode de vie urbain.

Pour autant, une partie de ces transformations n'aurait peut-être pas vu le jour sans la transition politico-économique. La diversification des économies locales demeure la clef de voûte de leur pérennité, car la question de la durabilité reste entière tandis que l'attachement des résidents s'illustre par les cottages. Les acteurs publics mènent des politiques d'encouragement de l'entreprenariat local. Mais les transformations observées, notamment des espaces de consommation et de récréation, ne sont pas propres à ces villes. Moscou, en tant que porte d'entrée du pays, de tête de pont vers les forces du marché, n'avait pas tant d'avance, la différence est plutôt en termes d'échelle du phénomène.

L'image appuie l'argumentation des changements illustrant la grande faculté d'adaptation des Russes. Le Huerou notait « les gens se sont adaptés : les pauvres à la pauvreté, les riches à la richesse... » (Le Huerou, 1998). Cette aptitude est démultipliée ici, comme sur tous les fronts pionniers, par une population jeune et dynamique capable de réinventer sa ville.

$\mathrm{Au}$ total, l'expérience de ces lieux, leur fréquentation ces vingt dernières années, conduisent à penser qu'il règne dans ces localités une certaine forme de convergence, traduisant une certaine normalisation, dans un monde global. Dans la zone arctique, les villes constituent des isolats où le désert s'interrompt, où la naturalité - la wilderness fait place à des paysages anthropiques de consommation et d'espaces privés loin des représentations communes de l'Arctique (Vaguet, 2016). Seul l'appareil productif était connecté jadis, rapportant des pétrodollars au pays et seule une toute petite fraction de l'élite locale pouvait éventuellement prétendre en profiter un peu. Aujourd'hui, le processus de mondialisation a percolé jusqu'à l'habitant et les différences socioéconomiques s'inscrivent dorénavant dans le paysage urbain. La mise en concurrence des villes soumet celles-ci à leur propre transformation pour répondre enfin aux attentes des résidents aussi après que l'industrie ait longtemps été prioritaire. Pour autant, certains demeurent exclus, notamment les néo-résidents et les travailleurs de quart logés dans les dortoirs.

Il resterait à analyser des différences infrarégionales entre ces villes, leur ancienneté, ainsi que la compagnie à laquelle elles sont attachées depuis leur naissance. Elles constituent peut-être des variables capables de les différencier en termes d'aptitude à se transformer vers le global. 


\section{BIBLIOGRAPHIE}

BAUDELAIRE C. (1863), Le peintre de la vie moderne.

BERQUE A. (2011), « Le rural, le sauvage, l'urbain », Études rurales, pp. 51-61.

BERTHOMIERE W. (2012), En-quête de signes : Migrations, Places et Continuité(s). Retour d'expérience à partir du cas israélien, thèse, Université de Poitiers, https://tel.archives-ouvertes.fr/tel-00979565/ document, accessed November 14, 2017.

BIAGGI C. (2015), « Habiter, concept novateur dans la géographie scolaire ? ", Annales de géographie, 704, pp. 452-465, 10.3917/ag.704.0338.

CHATEL C., MORICONI-EBRARD F. (2007), « Définir les villes nouvelles de Russie », Regard sur l'Est, http://www.regard-est.com/home/breve_contenu.php?id=779, accessed November 30, 2017.

COLLIGNON B. (2008), “'Urban life', 'Urbanity' : What is a City after all ? Geography's Basics on the Object 'Urban'”, in The Urban Arctic. Living Communities : New Perspectives on Inuit Urban Life, Copenhague, Eskimology and Arctic Studies Section/International PhD School for the Study of Arctic Societies (IPSSAS), pp. 9-16.

COUDROY DE LILLE L. (2016), « Entre Est et Ouest : une européanisation urbaine », Revue Géographique de l'Est, 56.

DE KONINCK R. (2000), “The theory and practice of frontier development : Vietnam's contribution”, Asia Pacific Viewpoint, 41, pp. 7-21, doi :10.1111/1467-8373.00103.

DEMANGEON A. (1932), « Pionniers et fronts de colonisation », Annales de Géographie, 41, pp. 631-636.

DYBBROE S. (2008), “Is the Arctic really urbanising ?", Études/Inuit/Studies, 32, 13, doi : 10.7202/029817ar.

DYBBROE S., DAHL J. \& MÜLLER-WILLE L. (2010), “Dynamics of Arctic Urbanization”, Acta Borealia, 27, pp. 120-124, doi : 10.1080/08003831.2010.527526.

ECKERT D. (2001), « Moscou : les mutations d'une capitale (1990-2000) », Revue Russe, 19, pp. 29-36, 10.3406/russe.2001.2093.

ECKERT D., JÉGOU L. (2004), « Evolution récente de la population de Russie (1979-2002) : cartographie interactive ", M@ppemonde, 74, 2, http://www2.cnrs.fr/sites/thema/fichier/ mappemonde74russieeckert.pdf, accessed November 29, 2017.

ESPIRITU A.A. (1999), The Impact of Industrialization and Resource Development on Indigenous Peoples of Northwest Siberia: The Khanty, Mansi and Iamalo-Nenets, Edmonton, University of Alberta, 223 p.

HELENIAK T. (2003), “The 2002 Census in Russia : Preliminary Results”, Eurasian Geography and Economics, 44, pp. 430-442, doi : 10.2747/1538-7216.44.6.430.

JURICEK J.T. (1966), “American Usage of the Word "Frontier” from Colonial Times to Frederick Jackson Turner", Proceedings of the American Philosophical Society, 110, pp. 10-34.

LATHAM A., MCCORMACK D.P. (2004), “Moving cities : rethinking the materialities of urban geographies”, Progress in Human Geography, 28, pp. 701-724, doi : 10.1191/0309132504ph515oa.

LE HUEROU A. (1998), « Russie : les paradoxes de la violence (Partie 2)», Cultures \& Conflits, doi : $10.4000 /$ conflits.694. 
LEES L. (2002), “Rematerializing geography : The 'new' urban geography”, Progress in Human Geography, 26, pp. 101-112, doi : 10.1191/0309132502ph358pr.

LOGUNOV E.V. (1999), Demographic and social consequences of oil and gas development in Siberia, Alaska, Universiy of Fairbanks, $264 \mathrm{p}$.

MARCHAND-VAGUET Y. (2005), « La conquête des hydrocarbures en Sibérie occidentale, le modèle centre-périphérie revisité », L’Espace géographique, 2, pp. 145-159.

MOLES A.A. (1978), « L'image et le texte », Communication et langages, 38, pp. 17-29, doi : 10.3406/ colan.1978.1199.

MONBEIG P. (1954), Le Brésil, PUF.

MONBEIG P. (1952), Pionniers et planteurs de São Paulo, Paris, A. Colin, 376 p.

MOTTA C. (2007), « L'expérience soviétique des villes nouvelles : évolution d'un paysage urbain », Regard sur l'Est, http://www.regard-est.com/home/breve_contenu.php?id=786, accessed November 30, 2017.

NOYABRSK (2017), АДМИНИСТРАЦИЯ ГОРОДА НОЯБРЬСКА, site officiel de la ville, http:// admnoyabrsk.ru/, accessed November 30, 2017.

RADVANYI J. (2010), La nouvelle Russie, Paris, Armand Colin.

REYNAUD A. (1981), Société, espace et justice : inégalités régionales et justice socio-spatiale, Paris, Presses universitaires de France, $263 \mathrm{p}$.

ROBEQUAIN C. (1952), «Une étude de front pionnier au Brésil de Mr Pierre Monbeig », Annales de Géographie, 61, pp. 458-460, doi : 10.2307/23442552.

SELLERON G. (2004), « Analyse d'un front pionnier par images satellitales multidates de 1987 à 2002. Le cas du nord-ouest du Petén (Guatemala) », Cybergeo : European Journal of Geography, 290, https://cybergeo.revues.org/3280, accessed October 3, 2017.

VAGUET Y. (2007a), Les hydrocarbures, les villes et les hommes dans le nord-ouest sibérien, Festival International de Saint-Dié des Vosges.

VAGUET Y. (2007b), « Pollution en Arctique », in CIATTONI A., VEYRET Y. (éd.), Géographie et géopolitique des énergies, Paris.

VAGUET Y. (2009), « Environnement, société et hydrocarbures dans le Grand Nord russe », Regard sur l'Est, http://www.regard-est.com/home/breve_contenu.php?id=911, accessed September 28, 2017.

VAGUET Y. (2013), "Oil and Gas towns in Western Siberia : past, present and future challenges", Proceedings from the First International Conference on Urbanisation in the Arctic, WP 6 :218, pp. 125-132.

VAGUET Y. (2016), More Urban, Less Arctic ? When Arctic Cities shape the Arctic Region, https:// www2.gwu.edu/ ieresgwu/assets/docs/Murmansk_Conference.pdf, accessed November 29, 2017.

VALENTINE G. (2001), Social geographies : space and society, New York, Prentice Hall, 400 p.

ZAITSEVA A.N. (2002), « La population de la province de Tioumen au XX $\mathrm{XX}^{\mathrm{e}}$ siècle », Espace Social (en russe), 20, pp. 65-70. 


\section{NOTES}

1. Les statistiques, bien que parcellaires, montrent plus de 1 million d'arrivées dans ces régions du Grand Nord russe entre 1959 et 1990 - il faut noter que les départs ont toujours été très nombreux aussi. Au total, entre 1959 et 2010, la population régionale est passée de 1,2 à 5,45 millions tandis que celle des deux districts autonomes augmentait de 0,186 à 2,05 millions et sa part passait ainsi de $15 \%$ à $38 \%$.

2. En analyse territoriale, elle s'appuie sur la matrice SWOT qui croise pour un territoire ses forces (Strengths en anglais), ses faiblesses (Weaknesses), ses opportunités (Opportunities) et ses menaces (Threats).

3. Mechta signifie 'rêve' en russe

\section{RÉSUMÉS}

Les hydrocarbures continuent de structurer la géographie et la géopolitique de notre monde. Néanmoins, les lieux d'extraction parfois à la limite de l'œcoumène et les modes de vie des résidents retiennent, somme toute, fort peu l'attention. La plaine de l'ob en constitue un excellent observatoire, dans la zone arctique et subarctique. Les villes pionnières pétrolières et gazières, toujours à la limite de la viabilité dans une économie désormais de marché, y enregistrent des transformations profondes depuis la chute de l'URSS. Tandis qu'elles avaient peu d'atouts pour les résidents et souffraient du désengagement de l'Etat, elles se sont ouvertes à la concurrence.

En adoptant une approche phénoménologique, il est possible de rendre compte et discuter des réalités matérielles des mutations de ces paysages urbains post-soviétiques. L'image et le texte se combinent pour mettre en évidence les changements opérés largement en raison de l'introduction de l'économie de marché et qui répondent aux aspirations des résidents. Déclinaison locale de la mondialisation? Une certaine forme de normalisation s'y observe : la ville pionnière devient une cité moderne.

Hydrocarbons continue to structure the geography and geopolitics of the world. Nevertheless, places of extraction sometimes at the limit of the ecumene, and the lifestyles of their residents draw little attention. The plain of the Ob river is an example in the Arctic and subarctic zone. The oil and gas towns there are at the frontier, they remain at the limit of viability in a market economy. They experience undergoing transformations since the fall of the USSR. They opened up to the competition whereas they had few assets for residents, and suffered from the withdrawal of the State.

The phenomenological approach allows to account for and discuss the material realities of the mutations of these post-Soviet urban landscapes. The image and the text combine to highlight changes as a result mainly of the introduction of the market economy, and that respond to the aspirations of residents. Some local forms of normalization are observed there, a result of the globalization declined locally. The frontier town becomes a modern city. 
INDEX

Keywords : Siberia, image, frontier town, post-Soviet, transformation, globalization, Russia, Ob Mots-clés : Sibérie, image, ville pionnière, post-soviétique, transformation, mondialisation, Russie, $\mathrm{Ob}$

\section{AUTEUR}

\section{YVETTE VAGUET}

CNRS UMR 6266 IDEES-Rouen, Université de Rouen (France), yvette.vaguet@univ-rouen.fr 